Estudios Románicos, Volumen 30, 2021, pp. 327-338

ISSN: 0210-4911

eISSN: 1989-614X

DOI: https://doi.org/10.6018/ER.471851

\title{
EL CICLO DE LA "VOLOMBRELLA" Y LOS LÍMITES DE LA TEMÁTICA NAPOLITANA EN EL CANSONERO DEL CONDE DE POPOLI
}

(The "Volombrella" cycle and the boundaries of Neapolitan topics in the Count of Popoli's Cansonero)

\author{
Francisco José Rodríguez-Mesa* \\ Universidad de Córdoba
}

\begin{abstract}
The Cansonero, whose compilation was ordered by Giovanni Cantelmo, Count of Popoli, is considered as the main testimony of the so-called "koine poetry", that emerges in Naples during the Aragonese period. According to its features, it could be said that this trend fluctuates between the courtly poetry and the popular and Meridional one. Maria Corti, one of the pioneers in the study of this anthology, stated: "l'elemento indigeno vive in promiscuità con cose e immagini che nel "400 erano ormai di dominio nazionale nella letteratura popolareggiante riflessa" (1956: XXVI). With these words she inaugurated a critical tendency which has been followed without any revision by most of scholars. This study intends to prove the validity of Corti's opinions on the basis of one of the most popular group of compositions in the Cansonero, the so-called "volombrella" cycle.
\end{abstract}

Keywords: Count of Popoli's Cansonero, koine poetry, popular poetry, barzelletta, volombrella, Aragonese Naples.

Resumen: El Cansonero encargado por Giovanni Cantelmo, Conde de Popoli, es considerado el máximo exponente de la denominada "lírica de koiné", surgida en Nápoles durante el dominio aragonés. Por sus características, se podría decir que esta vertiente oscila entre la poesía culta o cortesana y el filón popular de inspiración meridional. Una de las pioneras en el estudio de esta obra, Maria Corti, afirmó que, temáticamente, "l'elemento indigeno vive in promiscuità con cose e immagini che nel "400 erano ormai di

* Dirección para correspondencia: Francisco José Rodríguez-Mesa. Dpto. de Ciencias del Lenguaje. Área de Filología Italiana. Facultad de Filosofía y Letras. Plaza del Cardenal Salazar s/n. 14071, Córdoba. (francisco. rodriguez.mesa@uco.es) 
dominio nazionale nella letteratura popolareggiante riflessa" (1956: XXVI), inaugurando así una corriente de pensamiento crítico que ha sido aceptada sin discusión alguna por la mayoría de los estudiosos posteriores. En este estudio pretendemos comprobar la validez de las opiniones de Corti tomando como base uno de los grupos de composiciones más conocidos del Cansonero, el denominado ciclo de la "volombrella".

Palabras clave: Cansonero del conde de Popoli, lírica de koiné, poesía popular, barzelletta, volombrella, Nápoles aragonesa.

El comúnmente denominado Cansonero del conde de Popoli, que ha llegado hasta nosotros gracias al códice Ital. 1035 de la Biblioteca Nacional de Francia, está compuesto por un conjunto de poemas y algunas cartas reunidos por encargo de Giovanni Cantelmo, sexto conde de Popoli entre 1468 y 1478 aproximadamente. Tradicionalmente, el Cansonero se ha considerado el emblema de la denominada lírica de koiné, una vertiente de la poesía vulgar de moda en la corte napolitana de Ferrante de Aragón en las décadas de 1460 y 1470.

A diferencia de lo que ocurre con infinidad de etiquetas que la crítica ha utilizado para referirse a otros movimientos literarios, la denominación de "lírica de koiné" no se aplica a una categoría formada por composiciones homogéneas en cuanto a su temática o métrica. Así pues, tomando el Cansonero como paradigma, junto a poemas de temática popular (burlescos, antieclesiásticos, invectivas contra la mujer, etc.) hallamos una serie de composiciones amorosas cuyo tono corre paralelo al de otros poemas recogidos en cancioneros quattrocentescos de inspiración petrarquista. En el plano métrico encontramos una situación similar, pues con barzellettas, respetos o estrambotes convive un nutrido grupo de sonetos que, en no pocas ocasiones, se articula sobre la base de esquemas ya presentes en los $R v f^{1}$.

Ante esta situación, la lengua podría considerarse como el aspecto más homogéneo de toda la colección. No obstante, ¿de qué lengua se trataría? A pesar de los claros elementos de inspiración popular de algunas composiciones y de su temática netamente napolitana, no debe pensarse que el código lingüístico empleado en el Cansonero fuese una lengua viva más allá de la escritura. Por el contrario, se trata de un híbrido, una koiné que Maria Corti describió sirviéndose del algoritmo "dialetto + Petrarca + latino" (Corti 1956: XXXVI), elementos a los que, en ocasiones, habría que añadir el toscano quattrocentesco y el castellano y el catalán ${ }^{2}$ en cuanto lenguas presentes en la cotidianidad de la corte.

Como se puede imaginar, ante un fenómeno con unos componentes tan complejos y variados entre sí es difícil encontrar soluciones unívocas, de manera que las divergencias lingüísticas entre las rimas del Cansonero son significativas (vid. Rodríguez-

\footnotetext{
1 Uno de los mejores ejemplos de estos fenómenos -que ha pasado desapercibido tanto a Mandalari (1885) como a Altamura (1978) y a Gil Rovira (1991; 2007)- es la inclusión de una variante del soneto de Giusto de' Conti "Caro conforto a mie dolenti pene" (Bella mano CXLI, ed. Vitetti 1933) en Ital. 1035: el soneto "Dolce conforto de le mie ardente pene" (ff. 36v.-37r.), ya localizado por Vecce (2008: 303-304). Para consultar el conjunto de los sonetos del Cansonero así como su distribución dentro de él vid. Rodríguez-Mesa 2012: 186-198, 553-566.

2 Para más datos acerca del peso de estas lenguas ibéricas en la literatura napolitana del período aragonés, vid. Coluccia 1987 o Rovira (1990).
} 
Mesa 2016). Con todo, estas son mucho más tenues que las diferencias temáticas o métricas, puesto que en cada una de las composiciones es posible hallar -con mayor o menor dificultad- elementos que, lingüísticamente, remiten a un origen común. De hecho, Ital. 1035 es descrito en el catálogo de la Biblioteca Nacional de Francia como un "recueil de 141 poèmes rimés en dialecte napolitain"3.

A la vista de los variados componentes de este código lingüístico y de que la lengua supone el único elemento unitario de este filón poético, Corti bautizó esta corriente con la ya citada denominación de "lirica di koiné" (Corti 1956: XVI).

La vertiente de la lírica de koiné estaría formada, según Corti, por "due filoni di influsso popolare[:] l'indigeno, squisitamente napoletano, e quello legato nei suoi sviluppi a una tradizione da tempo interregionale e i cui frutti maturano in tutte le zone d'Italia" (Corti 1956: XX). En estas palabras hay varios elementos dignos de mención. En primer lugar, dadas las implicaciones ya expuestas de la denominación misma de lírica de koiné, los filones a los que hace referencia Corti han de ser forzosamente extralingüísticos, es decir, métricos o temáticos, puesto que las características lingüísticas de ambas categorías serán las de la koiné que da nombre al conjunto de la vertiente. En segundo lugar, al hablar de dos filones "d'influsso popolare", la estudiosa ignora el corpus de sonetos en cuanto representantes de una tendencia ajena al ámbito de la poesía popular. Este hecho, además, parece dar a entender que los dos filones a los que Corti alude no son, desde luego, de naturaleza métrica, puesto que los sonetos del Cansonero no son calificables métricamente como ejemplos de influencia popular. Por consiguiente, la naturaleza de estas dos corrientes que vertebran la lírica de koiné no puede ser más que temática.

Esta definición de Corti del Cansonero como una obra susceptible de ser reducida a dos filones populares ha gozado, paradójicamente, de un gran éxito entre los estudiosos posteriores de la obra. En efecto, no han sido pocos ni poco importantes los autores que han ignorado en sus consideraciones el eclecticismo temático y métrico de Ital. 1035 al dejar de lado la vertiente más áulica de la colección, ejemplificada en el conjunto de sonetos. Algunos ejemplos son los juicios de Tateo, que se refiere al grueso de la obra como materialización cumbre de una vertiente marcada por "modi popolareschi" (Tateo 1974: 110) o de Altamura, que describe el Cansonero como "poesia popolareggiante" (Altamura 1978: 14). Denominaciones como estas son válidas para con buena parte de las composiciones que forman la obra, pero se muestran problemáticas -cuando no directamente inaplicables-si se ponen en relación con el corpus de sonetos a los que se ha aludido.

Profundizando en la dicotomía de Corti, con respecto a la categoría del "elemento indigeno", la estudiosa asegura que "si distingue per un tono effervescente, folkloristicamente enfatico, raggiunto con formule di linguaggio popolare e richiami a maschere e simboli della tradizione napoletana" (1956: XX). En cuanto al filón de más amplia difusión geográfica, aduce que "frequentemente entro barzellette e strambotti ${ }^{4}$ 'elemento

3 http://gallica.bnf.fr/ark:/12148/btv1b8433318j [Última consulta 12/01/2021]. La cursiva es nuestra.

4 Nótese cómo, una vez más, Corti hace caso omiso a los sonetos presentes en el Cansonero. No obstante, esta omisión podría deberse al hecho de que Pietro Jacopo de Jennaro, poeta al que la estudiosa dedica su ensayo, no es autor de ninguno de estos sonetos. 
indigeno vive in promiscuità con cose e immagini che nel '400 erano ormai di dominio nazionale nella letteratura popolareggiante riflessa" (1956: XXVI).

También este juicio de Corti ha sido extremadamente influyente en los estudios posteriores sobre el Cansonero y, como prueba de ello, basta leer la opinión de Altamura sobre las divisiones internas de la obra:

È stato giustamente osservato che in questa lirica c'è un doppio filone popolare: quello tipicamente napoletano, con fatti e figure ed espressioni chiaramente locali, e un altro filone che si rifaceva a cose ed immagini che erano di dominio nazionale nella letteratura popolare riflessa. (Altamura 1978: 15).

Como decimos, esta doble división se ha aceptado como un axioma en un número significativo de estudios del Cansonero desde hace más de medio siglo, de modo que no se han revisitado los pasos que Maria Corti siguió para llegar a esta conclusión. En vista de este hecho, hemos decidido analizar de nuevo algunos de los textos que cita la estudiosa para comprobar hasta qué punto estos dos filones se manifiestan realmente en los poemas de Ital. 1035 y, principalmente, cuál es el verdadero alcance de la temática napolitana en esta obra. Puesto que las dos categorías establecidas por Corti son de influencia popular, dejaremos al margen de nuestras consideraciones el corpus de sonetos, para ceñirnos principalmente a las barzellettas.

Un grupo de composiciones interesante a la hora de analizar la pertenencia a uno u otro filón es el llamado ciclo de la "volombrella"; esto es, una tensó formada por tres barzellettas con estrambote, en la primera de las cuales $(5 a)^{5}$ se insta a una joven a que se entregue a los placeres del amor, mientras que la segunda (5b) y tercera (5c) recogen la respuesta de la dama. La primera de estas composiciones, situada entre 11r. y 11v., es obra de Pietro Jacopo de Jennaro; la segunda se encuentra entre 15v. y 16r., está precedida por el paratexto "Resposta dela bolonbrella", y justo después de ella comienza "L'altra resposta", tercera de las barzellettas, recogida entre 16r. y 16v. Estas dos últimas composiciones aparecen como anónimas en el códice, si bien han sido atribuidas a Coletta di Amendolea (Corti 1956: XXVII).

Con respecto a la relación que este ciclo mantiene con la dicotomía de los filones, Altamura afirma:

È ovvio che per noi esercitano una particolare suggestività quei componimenti in cui ci sembra di avvertire certi preannunzi di Velardiniello, del Basile, del Cortese ${ }^{6}$. Il più fresco e aggraziato è senza dubbio quello che canta di una riottosa ragazza paragonata alla "volumbrella", cioè a un fico immaturo. (Altamura 1978: 15)

\footnotetext{
5 Con respecto al ciclo de la "volombrella", nos serviremos de la numeración y del texto de Corti (1956: 14-18).

6 De nuevo, sentimos en Altamura el eco de las palabras de Corti: "la [...] vitalità [di alcuni testi] si può ricostruire attraverso [...] opere più tarde, come quelle di Velardiniello, del Basile, del Cortese, ecc.” (1956: XX).
} 
Ya Corti se refirió al ciclo de la "volombrella" para resaltar la diferencia estilística entre la barzelletta de De Jennaro y las dos respuestas a la misma. Tales consideraciones, sin embargo, van acompañadas de la afirmación de que este

motivo popolare, già magistralmente sfruttato da Lorenzo il Magnifico e dal Poliziano, dell'invito alla donna perché goda e faccia godere della sua labile giovinezza, introduce sì la gustosa variante napoletana del fico immaturo, buono per la breve stagione in cui è "mezzo ammollato", ma subito [De Jennaro] le conferisce riflessi stilistici e rime di un modello toscano. (Corti 1956: XXVII)

Centrándonos en lo que esta afirmación constituye respecto a la respuesta de Coletta -y dejando de lado, por el momento, la barzelletta de De Jennaro- volvemos a la encrucijada de partida: parece que, según Altamura, la lengua es elemento suficiente para justificar la pertenencia de una composición al filón indígena. Esta opinión se ve reforzada por el siguiente de los ejemplos expuestos, el estrambote que comienza "Segnora, per tuo amore me sfarria", donde asegura que es posible rastrear "[q]ualche influenza dialettale" (Altamura 1978: 15).

Tomando como base estas afirmaciones, en nuestra opinión, cabría indicar que Altamura no entiende del todo los principios de clasificación establecidos por Corti que, recordemos, parecen transcender la simple naturaleza lingüística, de modo que acaba simplificándolos y colgando la etiqueta de "tipicamente napoletano" a composiciones que de meridionales tienen pocos o ningún rasgo más allá de los estrictamente lingüísticos, que se dan por descontados en esta lírica de koiné.

Volviendo a las palabras de Corti sobre la "volombrella", desde nuestro punto de vista, parece exagerado calificar esta metáfora como una "gustosa variante napoletana" si, una vez más, pretendemos conferir a los filones una naturaleza que transcienda lo meramente lingüístico. Sin ningún género de dudas, la voz "volombrella", lingüísticamente hablando, es exclusiva del Mediodía italiano, pero en términos poéticos esto no debe considerarse como único indicador de pertenencia al filón indígena.

Es cierto, no obstante todo lo dicho hasta aquí, que todos y cada uno de los rasgos mencionados por Corti y Altamura confieren una componente de especificidad municipal a las composiciones, pero ¿son lo suficientemente importantes como para ser contrapuestos a un filón denominado de "dominio nazionale"? Para indagar una posible respuesta a esta cuestión veamos en qué términos se han referido los dos estudiosos a esta segunda vertiente.

El segundo de los filones, que se muestra deudor de corrientes poéticas presentes en el resto del territorio italiano, viene definido por Corti como una vertiente "legat[a] nei suoi sviluppi a una tradizione da tempo interregionale" (1956: XX), "anche se la loro lontana origine va ricondotta al Sud" (1956: XXVII). Entre los primeros ejemplos que la estudiosa cita de esta corriente se encuentra la barzelletta $6^{8}$, una peculiar cansone

7 La divergencia vocálica entre la nomenclatura de Corti y Altamura se debe a que la primera cita sirviéndose del término utilizado por la barzelletta de De Jennaro, "volombrella", mientras el segundo toma la palabra de la "resposta de la bolonbrella" (f. 15 v.).

$8 \quad$ Citamos aquí según la numeración de la edición de Corti. 
de partens $a^{9}$, pues no está dirigida a la amada, sino a un amigo. En este caso, Corti advierte de que "l'elemento tradizionale del linguaggio e la composizione delle immagini assumono un andamento unitario", subrayando que el texto está plagado de "violenti dialettalismi morfologici" (1956: XXX). Así, la ripresa y la primera estrofa de la canción rezan:

Parterrò, poi che mia sorte

vol ch'io parta a mmal mio grato,

col cor nigro e desperato

sempre mai chiamando morte.

Parterragio lamentando

la mia mala fortuna

e continuo biastimando

la sua cruda faccie bruna;

non s(erà) persona alcuna

chi mai m'abbia consolato,

col cor nigro e desperato

sempre mai chiamando morte.

Más allá de la reduplicación "a mmal" (v. 2), destaca, en el plano morfológico, la forma "parterragio" (v. 4), con la desinencia "-er(r)agio', típica del futuro en napolitano y que, además, se repite en modo anafórico al comienzo de la segunda estrofa.

Rasgos paralelos, aunque mucho más pronunciados en el plano fonológico, encontramos en el siguiente de los ejemplos aducidos por Corti, el de la barzelletta 11, de la que afirma que es digna de este puesto en el filón nacional debido a que se encuadra en el esquema tradicional que rige el género de la cansone de sdigno, de alcance suprarregional. Sin embargo, observa que De Jennaro no ha vacilado en esta composición a la hora de "abbandonarsi a confidenza con un linguaggio poco letterario, con una progressione di invettive che hanno il sapore gustoso di un ambiente che, per sfogo dei propri sentimenti, tenga in serbo ben altro che le ballate toscane" (Corti 1956: XXIX). Estos rasgos son fácilmente verificables observando la primera estrofa de la barzelletta:
Li toi sdigni sogno tanti
e llo pazziar che fai, che fastidio a li santi ne verria certo oramai;
tu te mustre altera assai
co 'sso tuo pompuso stare, io te farrò umilïare ancora che te pesa.

9 Es, de hecho, la pertenencia a este género el elemento que empuja a Corti a la clasificación en el filón nacional. 
Más allá del tono, vivazmente popular, cabe resaltar algunos fenómenos lingüísticos que inclinan la balanza de la koiné hacia el elemento indígena. Entre ellos, destacan la forma verbal "sogno" (v. 1), claramente meridional, la reduplicación sintáctica "e llo" (v. 2) o las formas metafonéticas "mustre" (v. 5) y "pompuso" (v. 6).

A pesar de todos estos rasgos lingüísticos, la autora destaca "il tono bilanciato fra l'ironia e la provocazione, in mezzo a un continuo crepitio dialettale della lingua" en los poemas, señalando que "l'autore sembra essere stato buon regista delle qualità enfatiche dello stile popolaresco" (Corti 1956: XXIX-XXX). Es decir, a pesar del potente componente lingüístico municipal que los textos encierran, son dignos de pertenecer al filón de dominio nacional por el modo en que, siendo composiciones que derivan de un género de difusión extrarregional, desarrollan su contenido.

Llegados a este punto, y sin olvidar el razonamiento que hasta aquí nos ha conducido, pasemos a observar las consideraciones que la estudiosa dedica al ciclo de la "volombrella". Dice Corti que la barzelletta 5 a de De Jennaro ha de considerarse heredera del filón de difusión nacional, porque, por un lado -como se citó más arriba-, responde a un motivo popular "già magistralmente sfruttato da Lorenzo il Magnifico e dal Poliziano" (1956: XXVII), y, por otro lado, porque su lengua incluye notables ecos toscanos, que resaltan inmediatamente si se comparan con la canción "Ben venga maggio" de Poliziano ${ }^{10}$. No obstante, en lo que tiene que ver con las respuestas $5 \mathrm{~b}$ y $5 \mathrm{c}$, a pesar de que siguen derivando de una tradición temática extrarregional, Corti justifica que deben excluirse de este filón por su "mescolanza di rozzo realismo e di sviluppo enfatico del motivo in chiave proverbiale, con rilievo di vocaboli extraletterari"11 (1956: XXVIII); es decir, precisamente por motivos que se adujeron -no considerándose relevantes- en el caso de las barzellettas 6 y 11 de De Jennaro.

Sigue llamando la atención la flexibilidad de estos criterios de clasificación a la luz de lo que Corti afirma en relación con otras composiciones de De Jennaro (1956: XXXXXXIII), juzgadas sobre la base de factores estrictamente temáticos, donde la lengua no parece desempeñar un papel relevante, como ocurre con el estrambote 7 o la barzelletta 9. De hecho, solo se vuelve a hacer alusión a la importancia del elemento lingüístico en relación con las barzellettas 4 y 10, que, según la crítica, han de ser clasificadas en el filón nacional a pesar de algunas "illegittime [...] aperture dialettali" (Corti 1956: XXXII).

Estos razonamientos llevan a Corti a afirmar:

Se De Jennaro si distingue positivamente fra gli altri poeti dell'antologia, ciò non avviene quasi mai per vigore $\mathrm{e}$ forza di immagini, bensì per una fedeltà da primo della classe ai modelli letterari e soprattutto per una notevole purezza linguistica. (Corti 1956: XXXII)

10 En esta comparación, como ya puso de relieve Corti (1956: XXVIII), destacan especialmente las coincidencias de las palabras en posición de rima.

11 Recordemos, como hemos indicado más arriba, que también Altamura (1978: 15) englobó la barzelletta $5 \mathrm{~b}$ en el filón indígena debido a lo dialectal de su lengua. 
Una postura similar es la que se mantiene con respecto a Cola di Monforte, cuya inclusión en este segundo filón se erige sobre la base de la 'toscanidad' de su lengua, que "contiene numerose dittongazioni non metafonetiche e segni dell'influsso toscano" (Corti 1956: XXXIV).

En nuestra opinión, tanto estos criterios de clasificación como su aplicación se revelan poco unitarios. Para comenzar, creemos que tendrían que responder a una naturaleza estrictamente temática, obviando cualquier tipo de consideración lingüística, como todas las que se han puesto de relieve en los ejemplos citados, pues de otro modo estaríamos ante una categoría redundante. El motivo que nos empuja a este razonamiento se fundamenta en la etiqueta misma de "lírica de koiné", en la que ya hemos profundizado: dado que todas estas composiciones se engloban bajo esta categoría que, como dijimos, es de naturaleza lingüística -y prueba irrefutable de ello es la misma alusión a la koiné a la hora de bautizar el filón- no hallamos pertinente llevar a cabo una subclasificación basada en la lengua o, al menos, condicionada por ella.

Aplicando el criterio que proponemos al ciclo de la "volombrella", no habría, por lo tanto, motivos para establecer distinción alguna entre la barzelletta de De Jennaro (5a) y las respuestas, pues temáticamente las tres están vinculadas a tópicos, como el del carpe diem, que trascienden con creces el ámbito meridional. Veamos algunos de los pasajes sobre la base de los cuales justificamos la pertenencia a este ámbito de dominio nacional:

Se la stagione ch'è si dolce e bella vene a passar, come vole natura, venendo da poi moscia e vecchiarella, assai te penterai de tua ventura; però t'ammolla e no essere sì fella, e laudarasse più toa fermosura, ca ciasche una fico volombrella a ffuria in quisto tempo se amatura. (5a, 21-28)

Si riputata so' formosa e bella, non divirissi dir che più amatura; Deo me faza esser semper volumbrella, chi cussì frisca tenga mia figura. Questa è la mia stagione tinirella, e non su', comu scrivi, fella e dura e veramente non ve inganna amore, ca so' più dulci dentro che de fore. (5b, 29-36)

Per quista cosa mi tengo contente, c'acerba mi trova omni mio inimico. Si mi remani lu cori e la mente, coll'arma santa e lu cori podico non mi curo di mari né di vente, 
né stimo troppo 'sti dissiti e dico:

bastami a mmi che io stipo a li denti

di mio marito questa prima fico. (5c, 21-28)

Naturalmente, ya en esta breve cita se hace patente la diferencia de tono que existe entre la composición de De Jennaro y las respuestas, divergencia a la que -como se ha repetido- ya aludieron Corti y Altamura. No obstante, para subrayar los principios que rigen nuestra clasificación, volvamos a la pregunta que anteriormente dejamos en el aire: ¿el elemento indígena es lo suficientemente significativo como para ser contrapuesto a un filón denominado de "dominio nazionale"? O, centrándonos en el ciclo de la "volombrella”, ¿hay algún elemento específicamente regional o indígena en 5b o 5c cuya aparición pudiese dificultar la transmisión del mensaje o de la intención poética más allá de las fronteras del Reino? En nuestra opinión no es así o, al menos, no lo es si se pretende operar en un plano puramente extralingüístico. Así pues, ambas réplicas a 5a persiguen una finalidad cómica al mostrar la respuesta de la dama a las observaciones iniciales de su interlocutor masculino. Este hecho, temáticamente, responde a una práctica poética consolidada desde tiempos remotos en una corriente ajena sur de la península italiana, y basta pensar en el contraste bilingüe de Raimbaut de Vaqueiras ("Domna tant vos ai preiada"), por citar un ejemplo concreto, para darse cuenta de ello.

Por otra parte, cabe destacar que todos y cada uno de los rasgos en que 5b y 5c suponen una ruptura para con 5a se podrían justificar sobre la base de la voluntad cómicorealista del autor. Así pues, el poeta de las réplicas podría haber modulado la lengua y, en general, el estilo de sus barzellettas para imitar el registro de la joven a la que da voz a través de ellas. Dicho de otro modo, cualquier manipulación que sitúa a 5 b y 5 c en una órbita estilística diversa a la de 5a podría ser consecuencia de la voluntad del autor por recrear, con la máxima verosimilitud posible, las palabras de la dama. No obstante, este tipo de procedimientos miméticos pueden atestiguarse desde lo más profundo de nuestra civilización literaria, por lo que, a nuestro juicio, no habría motivo temático como tampoco, por supuesto, métrico o lingüístico- para levantar esta barrera entre la barzelletta de De Jennaro y las atribuidas a Coletta.

Dejando de lado el contenido y centrándonos en un plano estrictamente textual, la clasificación de Corti se presenta igualmente conflictiva, y buen ejemplo de ello son los frecuentes elementos con función de deixis anafórica que unen las réplicas a la barzelletta de De Jennaro. Por ejemplo, son varias las estrofas de 5b y 5c (entre ellas la ripresa de ambos poemas) que comienzan con la conjunción condicional "si" (o sus variantes "se" o "s") seguida de una referencia a elementos de 5a. Así, la ripresa de 5b:

\section{Si a'stu tempo s'ammatura ogni frutto e ficocella io se so' pur volumbrella, è chi aspetta mia ventura}

remite a la de 5a: 
Fatte molla e non più dura

poi che si formosa e bella,

ché ogne fico volombrella

in chesto tempo se ammatura.

Del mismo modo, 5b, 9-12:

Lasse adunca a me culpare

se so' dura o tennerella,

io se so' pur volumbrella,

è chi aspetta mia ventura

5c, 5-6 ("Oi matura o acerba / che mi sia, quid a te?") y 5c, 21-22 ("Per quista cosa mi tegno contente, / c'acerba mi trova omni mio inimico") ${ }^{12}$ se basan en 5a, 5-8:

Fatte dolce e non più amara,

non te far tener più acerba;

per Dio, no essere più avara, fatte umile e non superba.

A la vista de estos hechos, que pueden probar una cierta unidad macrotextual más allá de las barzellettas aisladas, se podría decir que los deícticos son signos inequívocos de una red intratextual (más que intertextual), cuyos vínculos se mantienen firmes más allá de la ruptura estilística entre la composición de De Jennaro y sus réplicas.

No obstante, De Jennaro desempeña un papel fundamental en lo que a la vertiente del conjunto del ciclo se refiere. Así, este poeta, al determinar en la primera barzelletta de la tensó las características temáticas y estilísticas de su composición, inserta el conjunto del ciclo en un determinado filón, del que, recordemos, el autor de las réplicas no pretende desvincularse. Por consiguiente, si llegamos a la conclusión de que 5a debería englobarse en el filón de dominio nacional, carecería totalmente de sentido abogar por una distinta adscripción para $5 \mathrm{~b}$ y $5 \mathrm{c}$, ya que ambas composiciones son limitadamente autónomas y solo llegan a expresar totalmente su significado dentro del conjunto del ciclo. Proponer que 5a pertenezca temáticamente a un filón y que 5 b y 5 c remitan a otro conllevaría la fragmentación de la unidad en la que el ciclo se encuadra y gracias a la cual cada una de las barzellettas es capaz de materializar todas sus 'implicaturas' - por utilizar el término semántico-, de tal modo que se sacrificaría la mayor parte de los motivos cómicos, entre los cuales destaca el contraste en el registro lingüístico entre la composición de De Jennaro y las réplicas.

Aunque hasta ahora nos hemos referido fundamentalmente al ciclo de la "volombrella", por el mero hecho de que constituye uno de los principales textos a partir de los que Corti construye su dicotomía, la problemática que hemos expuesto es común al grueso del Cansonero, incluido el corpus de sonetos al que no se ha hecho referencia en estas páginas.

12 En todos los casos, las cursivas que muestran las deixis anafóricas son nuestras. 
Si bien la labor pionera de Maria Corti con respecto al Cansonero no deja de ser encomiable hoy en día, máxime si se tiene en cuenta la profundidad de sus reflexiones y su minuciosa descripción y edición de las composiciones atribuidas a Pietro Jacopo de Jennaro, se podría decir que todos los problemas o dificultades que entraña la clasificación de los poemas de Ital. 1035 como prototipo de la lírica de koiné remiten a dos motivos. En primer lugar, como se ha venido repitiendo, la misma aplicación de la etiqueta de "lírica de koiné" excluye cualquier intento de clasificación que pretenda basarse o, simplemente, tener en cuenta principios lingüísticos. En segundo lugar, centrándonos en la denominación de las categorías establecidas por Corti, en un momento de la historia de la literatura como el siglo XV ya ha comenzado a producirse en la península itálica un proceso de 'globalización cultural' tan profundo que buena parte de las temáticas que pueda manifestar cualquier tipo de obra poética estará lo suficientemente difundida geográficamente como para que se encuentren importantes escollos a la hora de pretender hacer remitir una determinada corriente a un filón estrictamente meridional. Asimismo, estas dificultades serán prácticamente insalvables si se parte del presupuesto de que una serie de "richiami a maschere e simboli della tradizione napoletana" (Corti 1956: XX) deberán ser los pilares sobre los que se eleve una especificidad municipal o regional con la suficiente entidad como para oponerse a un filón de dominio nacional.

En cualquier caso, creemos que las observaciones expuestas a lo largo de estas páginas prueban que se hace necesaria una revisión crítica de los presupuestos teóricos con los que la lírica de koiné se ha venido estudiando desde hace más de medio siglo.

\section{BIBLIOGRAFÍA}

AA.VV. Cansonero del Conte di Popoli, cód. Ital. 1035 de la Biblioteca Nacional de Francia [disponible en http://gallica.bnf.fr/ark:/12148/btv1b8433318j, 12/02/2021]. ALTAMURA, Antonio (1978): La lirica napoletana del Quattrocento. Napoli: Società Editrice Napoletana.

COLUCCIA, Rosario (1987): "Riflessi linguistici della dominazione aragonese nella produzione letteraria meridionale fra Quattro e Cinquecento", Giornale storico della letteratura italiana. Vol. 164: 57-69.

CORTI, Maria (1956): Pietro Jacopo De Jennaro. Rime e lettere. Bologna: Commissione per i testi di lingua.

GIL ROVIRA, Manuel (1991): El "Cansonero del Conte di Popoli", Ms. Ital. 1035 de la Biblioteca Nacional de París. Madrid: Tesis doctorales de la Universidad Complutense.

ed. (2007): Cansonero del Conte di Popoli. Madrid: Centro de lingüística aplicada Atenea.

MANDALARI, Mario (1885): Rimatori napoletani del Quattrocento. Caserta: Iaselli. RODRÍGUEZ-MESA, Francisco José (2012): "Qui risorga ogni laude del Petrarca": Petrarquismo y lírica culta en Nápoles hasta el ocaso de la dinastía aragonesa. Cór- 
doba: Tesis doctorales de la Universidad de Córdoba. [disponible en https:// helvia.uco.es/xmlui/bitstream/handle/10396/7621/571.pdf?sequence $=1$, 10/02/2021]

(2016): "Koiné, 'ma non troppo'. Apuntes acerca de la lengua de algunos autores presentes en el Cansonero del Conde de Popoli”, Scriptura. Vol. 23-25: 195-211.

ROVIRA, Juan Carlos (1990): Humanistas y poetas en la corte napolitana de Alfonso el Magnánimo. Alicante: Instituto de Cultura Juan Gil-Albert.

VECCE, Carlo (2008): "Echi contiani nella lirica aragonese", Italo Pantani (ed.), Giusto de' Conti di Valmontone. Un protagonista della poesia italiana del '400. Roma: Bulzoni, 297-315.

VITETTI, Leonardo, ed. (1933): Il canzoniere di Giusto dei Conti. Lanciano: Carabba.

\section{PERFIL ACADÉMICO Y PROFESIONAL}

Profesor Ayudante Doctor de Filología Italiana en la Universidad de Córdoba. Su investigación se centra en diversos aspectos de la literatura italiana medieval y renacentista, como el análisis de la lírica petrarquista y su difusión, en particular en el sur de la península italiana durante el Quattrocento (Giannantonio de Petruciis, Rustico Romano, el Cansonero del conde de Popoli), o Boccaccio y la tipología de las formas narrativas breves. Asimismo, se ha ocupado del papel de la mujer en algunas obras italianas del Trecento, principalmente en Boccaccio (Decameron, De mulieribus claris) y Petrarca (De insigni obedientia et fide uxoria, Rerum vulgarium fragmenta).

En los últimos años, ha impartido diversos seminarios en calidad de profesor visitante en varias universidades extranjeras, como la Università La Sapienza di Roma, la Università degli Studi di Udine o la Universidad Nacional Autónoma de México, y ha sido nombrado Investigador Asociado de la Biblioteca Nacional de Uruguay.

Fecha de recepción: 22-03-21

Fecha de aceptación: 02-05-21 